\title{
Non-Destructive Concrete Strength Evaluation Using PZT Based Surface Wave Propagation Technique - A Comparative Study
}

\author{
Kok Zee Kwong ${ }^{1, a}$, Yee Yan Lim ${ }^{2}$ and Willey Yun Hsien Liew ${ }^{3}$ \\ ${ }^{1}$ Civil Engineering Program, Faculty of Engineering, University Malaysia Sabah, 88400 Kota Kinabalu, Sabah, \\ Malaysia \\ ${ }^{2}$ Civil Engineering, School of Environment, Science and Engineering, Southern Cross University, Lismore NSW \\ 2480, Australia \\ ${ }^{3}$ Mechanical Engineering Program, Faculty of Engineering, University Malaysia Sabah, 88400 Kota Kinabalu, \\ Sabah, Malaysia
}

\begin{abstract}
Surface wave propagation (SWP) technique employing Lead Zirconate Titanate (PZT) transducer has recently been found to be a useful tool in concrete hydration monitoring. In this paper, the performance of PZT based SWP technique was compared with the conventional techniques such as ultrasonic pulse velocity (UPV) test, rebound hammer (RH) test and concrete compression test. Results showed that the SWP technique, in addition to its inherent advantages, performed equally well as the conventional counterparts in concrete strength prediction.
\end{abstract}

\section{Introduction}

Structural safety is the ultimate concern of engineering design. Failure due to insufficiently cured concrete during construction as well as concrete weakening from aging and deterioration occurred every now and then. As a result, an effective concrete strength monitoring technique is highly sought after to ensure safety and cost effectiveness.

A great variety of conventional techniques are available in the market for the monitoring of concrete strength development and continuous health assessment of concrete. These techniques are broadly classified into destructive evaluation technique and non-destructive evaluation (NDE) technique. Destructive evaluation techniques usually involve core test and loading test. These techniques induce damage to the existing structure as they require extraction of cylindrical specimens from the structure, which is often undesirable. On the other hand, NDE techniques include resonant frequency technique, hydration heat-based monitoring technique, ultrasonic pulse velocity (UPV) test and rebound hammer $(\mathrm{RH})$ test.

Recent development of smart material, namely piezoelectric (Lead Zirconate Titanate, PZT) transducer is capable in overcoming some shortcomings of the conventional structural health monitoring (SHM) techniques. Initially, the PZT transducer is used primarily for damage monitoring involving thin metallic or composite structures. It has later found its application in concrete hydration

\footnotetext{
${ }^{a}$ Corresponding author : charleskwongkz@gmail.com
} 
and strength monitoring. The advantages of employing PZT transducer based SHM techniques include autonomous, continuous and online remote monitoring, low power consumption and cost effective.

Surface wave based concrete hydration monitoring using conventional techniques has been well established. For instance, Luo and Bungey introduced a method to determine the dynamic modulus of elasticity for concrete using compression wave [1]. Moreover, Song et al. conducted a study on SWP in concrete structures using a PZT actuator/sensor system [2]. To date, research on WP techniques focused mainly on SHM of metallic and composite structures [3-5].

PZT based SWP technique has shown its capability in predicting the dynamic Young's modulus [6] and compressive strength of concrete [7]. This study aimed at comparing the performances of different concrete strength monitoring techniques, namely the PZT based SWP technique, UPV test, RH test and compression test. Study was conducted on a series of lab-sized mortar specimens with different strength.

\section{Physical Principle of Wave Propagation}

In SWP technique, two or more PZT transducers are permanently bonded on the surface of concrete specimen using high strength epoxy at predetermined distances. One of the patches is assigned as actuator to excite a five-peak tone burst signal. Another patch acts as a sensor to pick up the wave from the actuator. In this study, actuation of PZT transducer on the surface of thick concrete structure is expected to predominantly excite surface wave (Rayleigh or R-wave). R-wave propagates mainly along the surface of the concrete and decreases rapidly in magnitude with depth below the surface [8]. Weaker pressure wave (P-wave) may also be present [9].

It is noteworthy that the P-waves are characterized with high velocity and low amplitude, while the R-waves have lower velocity and higher amplitude [10]. Therefore, the first wave packet is induced by the propagation of $\mathrm{P}$-wave while the second wave packet is induced by $\mathrm{R}$-wave as shown in Figure 1. Waves following these two wave packets are complex reflections of bulk waves and noises.

Velocity of P-wave and R-wave can be calculated by measuring the time of flight (TOF) for any distance between actuator and sensor. TOF is the time calculated from wave packet of the actuator to the arrival of the wave packet of the sensor (Figure 1). As the concrete hardens, it becomes stiffer and thus affecting the velocity of the wave. Consequently, the TOF of the wave which can be measured from the electrical signatures, is altered. The data acquired is analyzed using various signal processing techniques such as Hilbert transform and cross-correlation analysis to achieve higher accuracy.

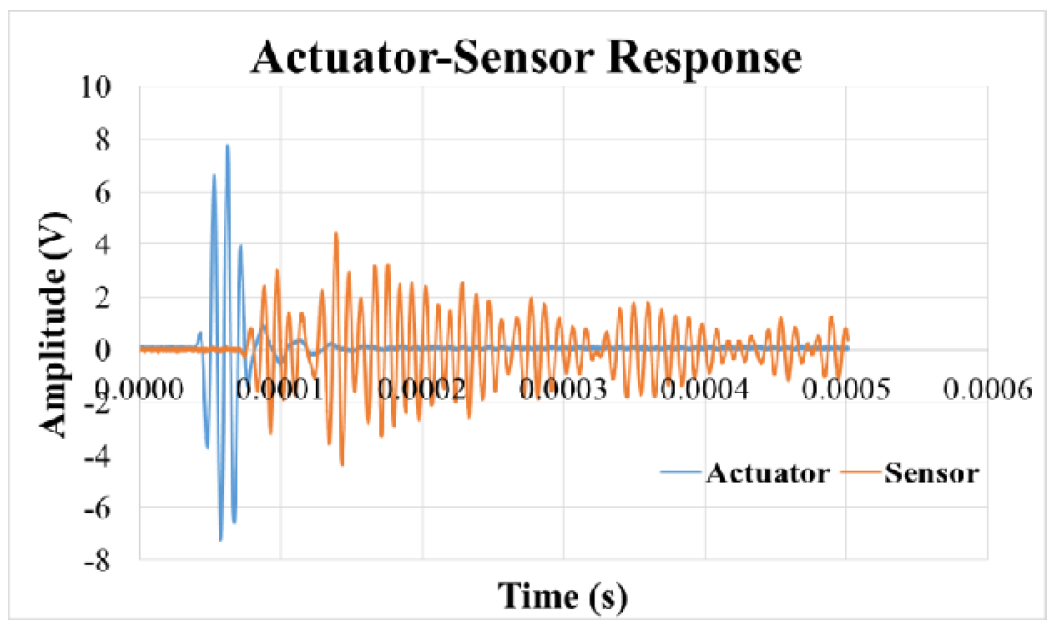

Figure 1. Wave packet and TOF for Prism $\mathrm{H}$ at $120 \mathrm{kHz}$ and $120 \mathrm{~mm}$ actuator-sensor distance. 
The fundamental theories of WP techniques were described by Jones [11]. The relationship between the modulus of elasticity of the solid medium and the wave velocity in homogeneous and isotropic solids of infinite dimensions can be expressed as:

$$
\begin{gathered}
V_{P}=\sqrt{\frac{E_{d}(1-v)}{\rho(1+v)(1-2 v)}} \\
V_{R}=\frac{0.87+1.12 v}{1+v} \sqrt{\frac{E_{d}}{\rho} \frac{1}{2(1+v)}}
\end{gathered}
$$

where, $E_{\mathrm{d}}$ is the dynamic modulus of elasticity, $v$ the Poisson's ratio, $\rho$ the density, $V_{\mathrm{P}}$ the velocity of pressure wave, $\mathrm{V}_{\mathrm{R}}$ the velocity of surface wave.

\section{Experimental Setup}

Two batches of cement mortar were mixed using cement to sand ratio of 1:2. First batch of cement mortar (Prism H) was mixed with water to cement ratio (W/C ratio) of 0.3 . The second batch of cement mortar (Prism L) was mixed with W/C ratio of 0.4 . Two cement mortar prisms with the dimension of $100 \mathrm{~mm} \times 100 \mathrm{~mm} \times 500 \mathrm{~mm}$ were prepared for each batch of casting. Cement mortar cubes with the dimension of $100 \mathrm{~mm} \times 100 \mathrm{~mm} \times 100 \mathrm{~mm}$ were also prepared from the same batch of mix for the evaluation of compressive strength after 1 day, 3 days, 7 days, 14 days and 28 days of curing. Three pieces of PZT patches with the dimension of $20 \mathrm{~mm} \times 20 \mathrm{~mm} \times 0.2 \mathrm{~mm}$ (PI ceramics) as shown in Figure 2 were surface bonded using two parts quick set epoxy onto the smooth surface of Prism $\mathrm{H}$ and Prism L respectively. The distances between Patch 1 and Patch 2, and Patch 2 and Patch 3 were $80 \mathrm{~mm}$ and $120 \mathrm{~mm}$, respectively as depicted in Figure 3.

A Waveform Generator (Agilent 33500B Series) was used to generate a $10 \mathrm{~V}$ peak-to-peak Hanning windowed five-peak tone burst at a frequency of $120 \mathrm{kHz}$. Due to the high rate of energy dissipation in concrete, the signal input on the actuation must be strong enough to generate appreciable output from the sensors. The signal was amplified through a Power Amplifier (Trek PZD350A) to $150 \mathrm{~V}$ peak-to-peak which was then applied to the actuating PZT patch. The signal from the sensor was measured using a Digital Oscilloscope (YOKOGAWA DL 1620) and recorded on a personal computer. The setup is schematically presented in Figure 4.

For both specimens, Patch 1 was first actuated, and this was followed by Patch 2 and Patch 3 acting as sensors. Patch 2 was later actuated with Patch 3 acting as sensor. Readings were taken immediately after the setting of epoxy and monitored continuously for the first 7 days and thereafter at 14 days, 21 days and 28 days. UPV, rebound hammer and compression tests were performed at 1 day, 7 days, 14 days, 21 days and 28. Both specimens (Prism H and L) were subjected to the same compaction and curing condition. The cubes and samples were cured outdoor under a roof, simulating site curing condition.

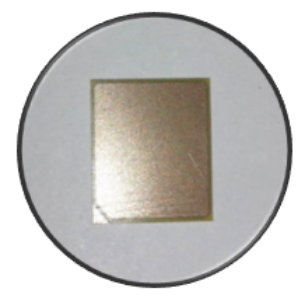

Figure 2. PZT patch. 


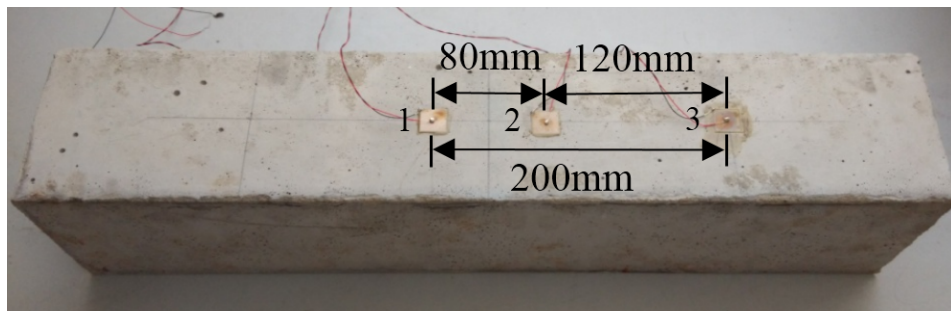

Figure 3. Configurations of PZT patches.

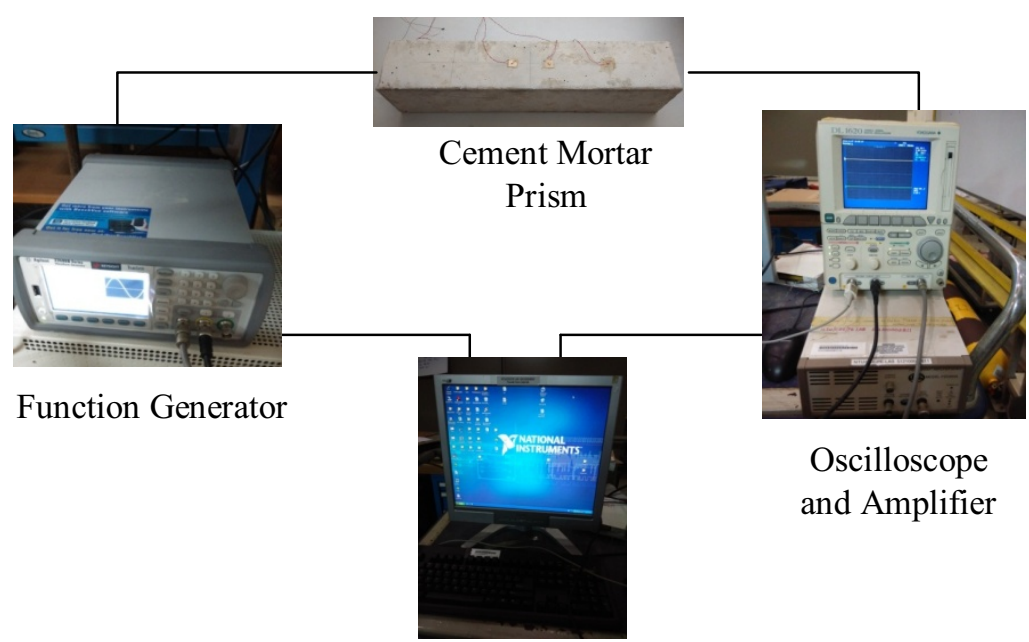

Computer

Figure 4. Experimental setup.

\section{Results and Discussions}

The sensor signal obtained from early age of curing (less than 9 hours) was very weak for both Prism $\mathrm{H}$ and Prism L indicating that the concrete was in a "soft" and "semi-solid" state. High damping and low stiffness prevented the P-wave and R-wave from reaching the sensor effectively. Moreover, the sensor signals acquired were seriously contaminated by noise. As a result, the sensor signal's TOF for P-wave and R-wave could only be measured after 19 hours of curing.

Figure 5 illustrates the relationship between the wave velocity and the curing time for P-wave and $\mathrm{R}$-wave, respectively. The results showed that all velocities increased with curing time. According to the elastic wave theory, the velocity of wave is proportional to the stiffness of the medium. In this case, as the concrete hardened, and its stiffness and strength increased, these was reflected in the increase in the velocity. On the other hand, the P-wave velocities and R-wave velocities acquired from Prism $\mathrm{H}$ were consistently higher than those from Prism L. This indicated that the stiffness and the strength of Prism H was higher than Prism L. 


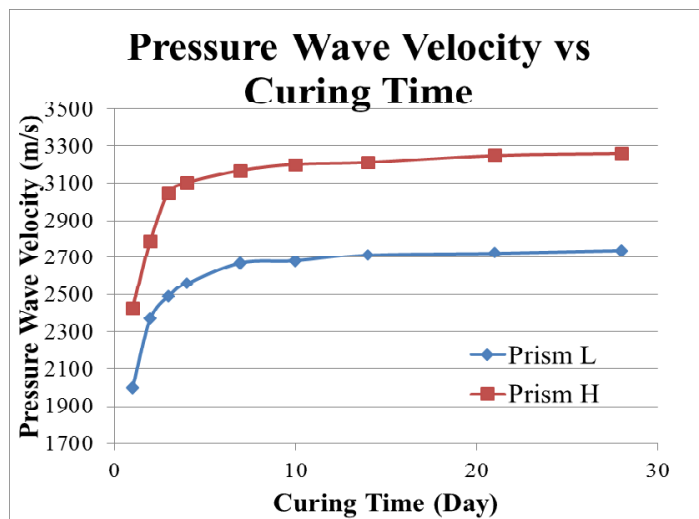

(a) Pressure wave

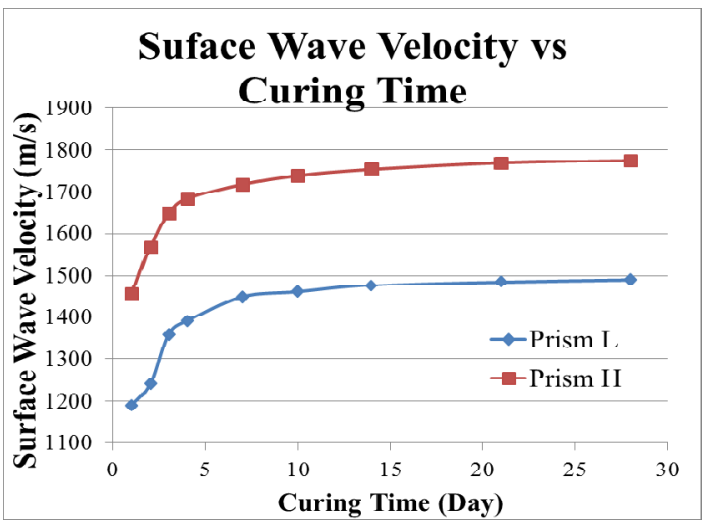

(b) Surface wave

Figure 5. Wave velocity versus curing time.

Knowing the velocities of P-wave and R-wave, the dynamic modulus of elasticity and Poisson's ratio can be evaluated using Equation 1 and Equation 2. The ACI equation was widely adopted in the engineering community to empirically relate the dynamic modulus of elasticity and the compressive strength [12]:

$$
E=k \sqrt{f_{c u}}
$$

where $E$ is the Young's modulus of concrete, $f_{\text {cu }}$ the compressive strength of concrete, $k$ the curve fit proportionality constant.

Straight line curve fitting was plotted using the least square method from relationship between dynamic Young's modulus of concrete and square root of concrete strength. The gradients for the straight line are the P-wave velocity and R-wave velocity. In this study, $k$ value was found to be 3.24 for both Prism $\mathrm{H}$ and $\mathrm{L}$.

Combining Equation (2) and Equation (3), the concrete strength can be correlated to the R-wave velocity through a semi-empirical relationship as presented schematically in Figure 6:

$$
f_{c u}=\left[\frac{2 \rho(1+v)\left(\frac{V_{S}(1+v)}{0.87+1.12 v}\right)^{2}}{k}\right]^{2}
$$

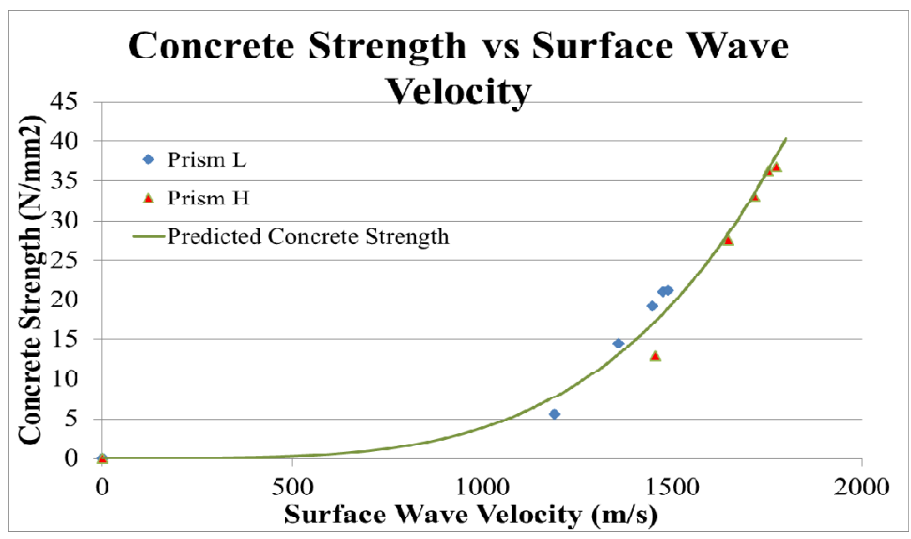

Figure 6. Strength prediction chart (SWP technique). 


\section{Comparison with Conventional Techniques}

Figure 7 shows the comparison of the concrete strength obtained from the SWP technique, and the compression, rebound hammer and UPV tests. In general, the rebound hammer test yielded to a more conservative outcome for both specimens. Most of the predicted concrete strength were lower than the actual concrete strength obtained from the compression test. The standard deviation with the concrete strength obtained from compression test is 4.38 .

The predicted strength obtained from the SWP techniques and UPV was similar, indicating that both techniques work equally well. They also showed superiority over the Rebound Hammer test in terms of accuracy of prediction. The standard deviation for UPV is 1.83 while the standard deviation for SWP technique is 2.66 .

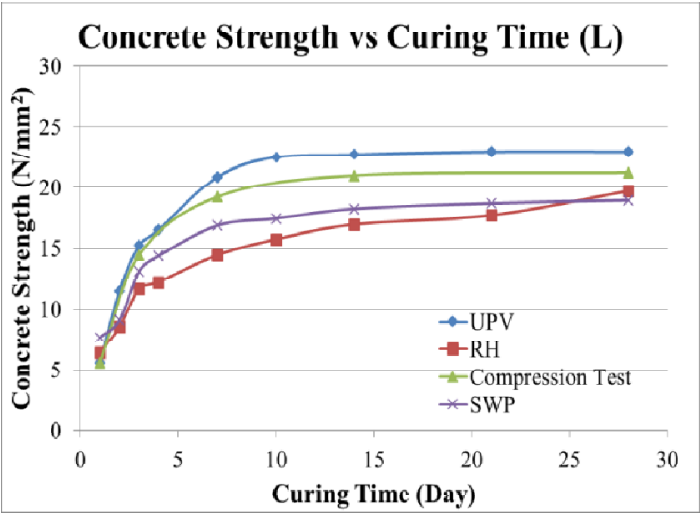

(a) Prism L

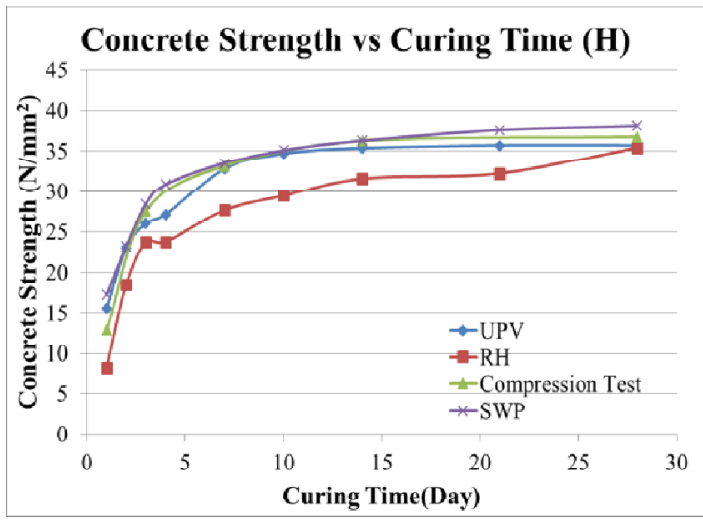

(b) Prism $\mathrm{H}$

Figure 7. Concrete strength versus curing time.

\section{Conclusions}

This paper presents a comparative study into the concrete strength prediction capability of SWP technique and similar conventional techniques, namely the UPV, the rebound hammer and the compression tests. It was shown that the SWP technique has the capability of predicting the strength of different types of concrete. It also performs equally well as the UPV test, yielding reasonably accurate predictions as compared to the compression test.

SWP technique enables users to measure both the R-wave and P-wave simultaneously. Hence, both the dynamic Young's modulus and Poisson's ratio of concrete can be evaluated.

As the results are promising, SWP technique could potentially replace the conventional NDE counterparts in providing an autonomous, real-time and remote concrete strength prediction technique. Future studies should focus on the investigation of the consistency, repeatability and reliability of this technique as well as the FE modelling on the PZT based SWP technique. On the other hand, a handy list of correction/modification factors accounting for varying conditions (e.g. temperature, humidity, curing condition, surface roughness, presence of aggregate, size of aggregate, types of cement etc.) should be established before real life application becomes possible.

\section{References}

[1] Q. Luo and J.H. Bungey, Using compression wave ultrasonic transducers to measure the velocity of surface waves and hence determine dynamic modulus of elasticity for concrete, Journal Construction and Building Materials, 10,237-242, (1996). 
[2] F. Song, G.L. Huang, J.H. Kim and S. Haran, On the study of surface wave propagation in concrete structures using a piezoelectric actuator/sensor system, Journal Smart Material and Structure, 17(5), 1-8, (2008).

[3] V. Giurgiutiu, J. Bao and W. Zhao, Active sensor wave propagation health monitoring of beam and plate structures, Proceeding SPIE's 8th Annual International Symposium on Smart Structures and Materials, 234-245, (2001).

[4] M. Gresil and V. Giurgiutiu, Guided wave propagation in carbon composite laminate using piezoelectric wafer active sensors, Proceeding SPIE Smart Structures and Materials+ Nondestructive Evaluation and Health Monitoring, 869525-869525, (2013).

[5] J.B. Ihn and F.K. Chang, Detection and monitoring of hidden fatigue crack growth using a builtin piezoelectric sensor/actuator network: I. Diagnostics, Journal Smart Material Structure, 13, $609,(2004)$.

[6] Y.Y. Lim, N. Majain, K.Z. Kwong and W.Y.H. Liew, On the study of concrete hydration process using piezoelectric based surface wave propagation technique, ACMSM23, 1121-1128, (2014).

[7] Y.Y. Lim, K.Z. Kwong, W.Y.H. Liew and C.K. Soh, A feasibility study into concrete strengthprediction using PZT based surface wave propagation technique, Journal NDT\&E International, (2014).

[8] Y. Lu, J. Li, L. Ye and D. Wang, Guided waves for damage detection in rebar-reinforced concrete beams, Journal Construction and Building Materials, 47, 370-378, (2013).

[9] L. Yu, In-situ structural health monitoring with piezoelectric wafer active sensor guided-wave phased arrays, PhD Thesis, University of South Carolina, (2006).

[10]M. Sun, W.J. Staszewski, R.N. Swamy and Z. Li, Application of low-profile piezoceramic transducers for health monitoring of concrete structures, Journal NDT\&E International, 41, 589595, (2008).

[11]R. Jones, Non-Destructive Testing of Concrete, Cambridge University Press, London, (1962).

[12] ACI Committee: 318-1995 Building code requirements for structural concrete and commentary, American concrete Institute, Michigan, (1995). 